\title{
We Asked the Experts: Breaking the Cycle of Attrition in Perioperative Academic Medicine
}

\author{
Catherine L. Chen ${ }^{1,2} \cdot$ Jennifer M. Lucero ${ }^{1,3}$
}

Published online: 26 June 2020

(C) Société Internationale de Chirurgie 2020

\section{Introduction}

There is increasing awareness that a diverse healthcare workforce is essential for improving the nation's health outcomes [1-3]. In response, academic medical centers have focused their efforts on promoting gender equity and inclusion, as well as implementing policies to support the retention and advancement of trainees and faculty who have historically been underrepresented in medicine (UIM) [2-4]. The value of these efforts is seen in studies that demonstrate that surgical outcomes are improved when the perioperative specialties reflect a diverse workforce [5]. Unfortunately, diversity initiatives at academic medical centers do not automatically translate into clinical leadership and executive management positions, especially in the perioperative specialties (Fig. 1) [1]. According to the AAMC [6], 31\% of US medical school graduates and a weighted average of $29 \%$ of graduating residents and $31 \%$ of assistant professors in the combined fields of anesthesiology, obstetrics/gynecology, general surgery, and surgical subspecialties identify as UIM or Asian. However, the prevalence of UIM or Asian faculty drops by nearly $40 \%$ among full professors and almost $50 \%$

Jennifer M. Lucero

jennifer.lucero@ucsf.edu

1 Department of Anesthesia and Perioperative Care, UCSF, San Francisco, CA, USA

2 Philip R. Lee Institute for Health Policy Studies, UCSF, San Francisco, CA, USA

3 Department of Obstetrics, Gynecology and Reproductive Sciences, UCSF, San Francisco, CA, USA among department chairs, indicating that faculty of colorparticularly female faculty of color-have yet to achieve parity in perioperative academic leadership. Below, we highlight two mechanisms that contribute to the persistence of racial disparities in academic leadership for the perioperative specialties: institutionalized barriers and personally mediated barriers [7].

\section{Institutionalized barriers}

Institutionalized barriers are policies, procedures, or situations that systematically disadvantage certain groups of people [7]. An example is when faculty of color are not aggressively recruited to lead clinical services or placed on high-level search committees for key leadership positions because they themselves do not already hold a senior faculty rank. These barriers are also present when perioperative faculty (irrespective of race or gender) are overlooked for extra-departmental leadership positions because of the predominance of faculty from the non-perioperative specialties who tend to nominate their own colleagues for these positions. Finally, a broader lack of oversight and accountability for achieving diversity metrics within perioperative departments often perpetuates the cycle of attrition.

\section{Personally mediated barriers}

Personally mediated barriers are present when assumptions or stereotypes about the perceived abilities of other races result in differential actions toward an individual 


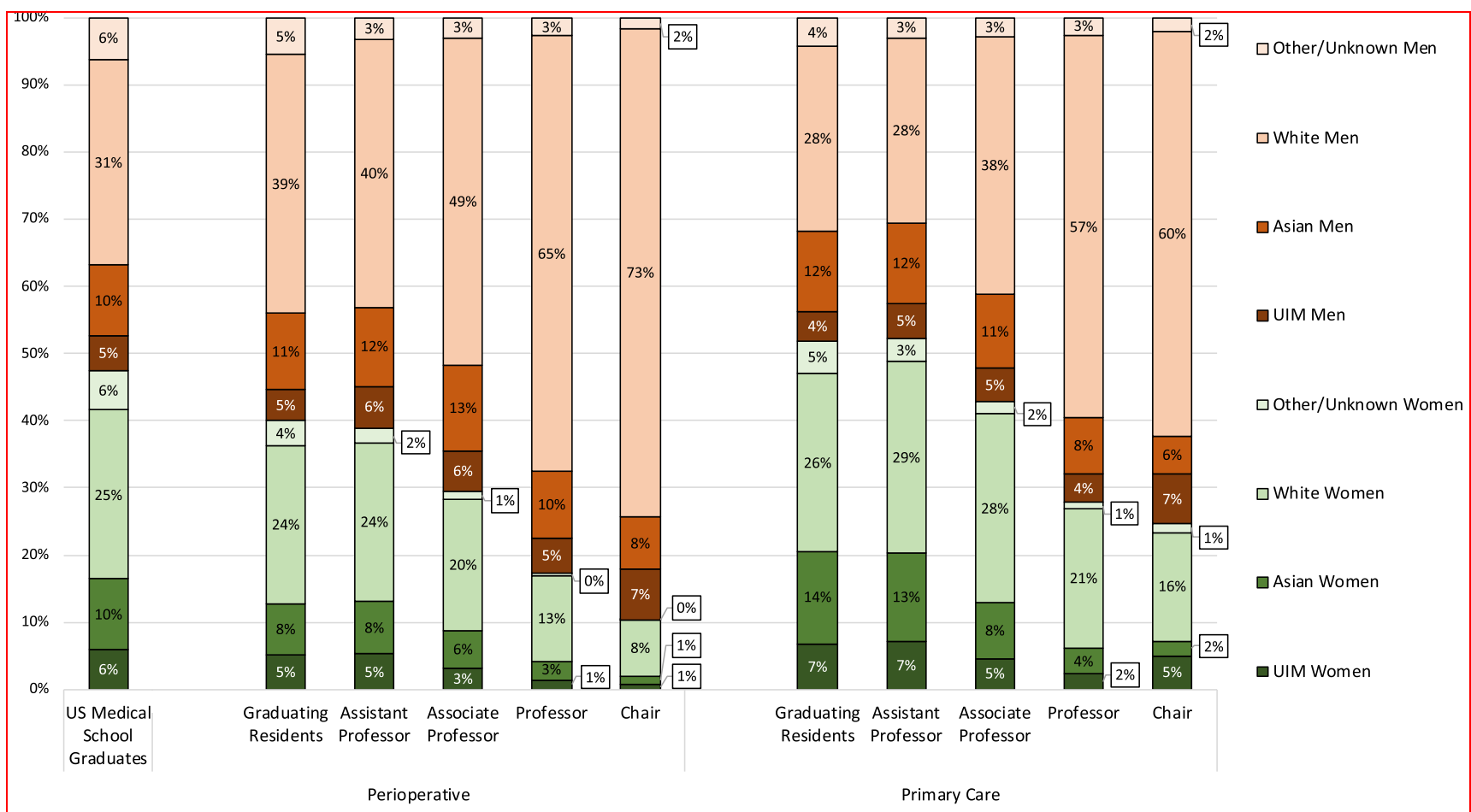

Fig. 1 Academic rank stratified by race and gender, 2017-2018 (see Appendix)

based on race [7]. When current perioperative healthcare leaders discuss the reasons that diversity efforts have not yet succeeded, they often state one or more of the following:

- Our specialty doesn't attract people of color; they want to work in primary care and give back to their community.

- We want diversity, but we also want qualified people.

- He/she just wasn't the right fit for our department.

- I am colorblind; I just try to choose the best person for the job.

These statements exemplify the indirect and subconscious racial biases held by people who might otherwise regard themselves as nonprejudiced, a concept known as aversive racism [8]. Current perioperative leaders unwittingly use these types of statements to rationalize the lack of diversity at every academic rank, especially at the most senior levels due to preexisting notions about what constitutes a leader. Although these statements are not intended to hamper faculty of color, they unfortunately have the same impact as traditional, overt racism in limiting the advancement opportunities available to UIM and Asian faculty. These prevailing attitudes and institutional practices must be challenged and solutions enacted.

\section{Role of sponsorship}

Although perioperative departments often commit significant resources to recruit, retain, and mentor faculty of color, these efforts are not sufficient to transform the diversity of the perioperative leadership. To perpetuate change, current leaders must also embrace the critical role of sponsorship for nurturing successful careers. Sponsorship is distinct from mentorship in that a sponsor typically holds a position of power and influence and can pledge their reputation and credibility to advocate for specific individuals when appropriate career and leadership opportunities arise. Importantly, sponsors must be willing to persuade their peers to consider perioperative faculty of color who may otherwise be overlooked. While strong sponsorship is necessary for faculty of color at all academic ranks, at the associate professor level, sponsorship directly leads to the next phase of career advancement by unlocking access to suitable leadership roles.

\section{Future}

Our primary care colleagues have been intentional and effective in their efforts to develop diverse leaders [9]. Using some of the same principles put forth by internal 
medicine chairs over a decade ago [9], we propose the following action steps to accelerate the establishment of a truly diverse and inclusive perioperative leadership structure:

- All perioperative faculty should be required to undergo formal training in implicit bias.

- Every perioperative department should recruit or appoint a Vice Chair of Diversity and Inclusion who is a person of color.

- The Department Chair and the Vice Chair of Diversity and Inclusion should meet annually with each faculty of color to discuss career goals, identify senior mentors and sponsors within and outside the department, and review immediate and long-term career advancement and leadership opportunities.

- Every executive search committee should include at least one member who is a person of color.

- When a departmental or executive healthcare leadership position becomes available, the executive search committee should encourage all associate and full professors of color to apply and should consider these candidates in earnest.

- Faculty of color who may normally be dismissed as "too junior" or "too inexperienced" to fill an open leadership position should be considered to have the same potential for success and be equipped with the same resources to achieve success as white faculty with equivalent experience.

- When faculty of color leave the university for a nonacademic position or a lateral position at another academic institution, this should prompt a detailed review by an extra-departmental committee to determine the underlying causes and enact solutions to avert future attrition under similar circumstances.

\section{Conclusion}

Academic medical centers are at the forefront of caring for our most vulnerable populations and training our future healthcare leaders. We must work at the highest levels to create a diverse, equitable, and inclusive environment to reduce disparities and improve the care of surgical patients. Diversifying medical students, trainees, and junior faculty will not guarantee a pipeline of diverse perioperative leaders unless it is clear to trainees that there is a path forward. All perioperative departments must be intentional about promoting and sponsoring men and women of color to institute effective change in the diversity of our perioperative leadership. We urge perioperative leaders to review their diversity metrics at all faculty ranks and consider enacting the changes outlined above to continue diversifying the face of perioperative academic leadership.

\section{Appendix}

1. The category, "Perioperative," includes Departments of Anesthesiology, Obstetrics/Gynecology, Ophthalmology, Otolaryngology, Orthopedic Surgery, and Surgery as listed in the AAMC Faculty Roster. The AAMC Faculty Roster groups all other surgical specialties not explicitly listed on the AAMC Faculty Roster into the "Surgery" category.

2. The category, "Primary Care," includes Departments of Internal Medicine, Family Medicine, and Pediatrics as listed in the AAMC Faculty Roster.

3. Specialties at the resident rank were categorized to be consistent with the specialty categories listed in the Faculty Roster.

4. "UIM" is an acronym for underrepresented in medicine and includes the following male and female categories at all academic ranks: American Indian or Alaskan Native, Black or African American, Hispanic, Latino or of Spanish Origin, Native Hawaiian or Other Pacific Islander. UIM at the faculty and chair rank also includes individuals categorized as "Multiple RaceHispanic." The "Other/Unknown" category includes "Multiple Race" at the medical student and resident ranks, "Multiple Race-Non-Hispanic" at the faculty and chair ranks, and "Other" or "Unknown" at every rank. Non-U.S. citizens and non-permanent residents at the medical student and resident ranks were excluded.

5. The weighted average percentage of each race and gender category at each academic rank was determined by calculating the combined race and gender percentages for each specialty multiplied by the frequency weight (i.e., the proportion of individuals within each specialty included in the "Perioperative" or "Primary Care" categories at each rank, respectively), then summing the weighted percentages for each combined gender and race category within each rank.

6. 2018 medical school graduate data was obtained from the AAMC Facts Table at https://www.aamc.org/ system/files/2019-11/2019_FACTS_Table_B-4.pdf. A custom data report for 2017 residency graduates categorized by specialty, race and gender was obtained directly from the AAMC. Faculty data was obtained from the 2018 AAMC Faculty Roster at https://www. aamc.org/system/files/2020-01/2018Table19.pdf. 


\section{References}

1. Abelson JS, Wong NZ, Symer M, Eckenrode G, Watkins A, Yeo HL (2018) Racial and ethnic disparities in promotion and retention of academic surgeons. Am J Surg 216:678-682

2. Newman EA, Waljee J, Dimick JB, Mulholland MW (2019) Eliminating institutional barriers to career advancement for diverse faculty in academic surgery. Ann Surg 270:23-25

3. Diaz T, Navarro JR, Chen EH (2020) An institutional approach to fostering inclusion and addressing racial bias: implications for diversity in academic medicine. Teach Learn Med 32:110-116

4. Thompson-Burdine JA, Telem DA, Waljee JF et al (2019) Defining barriers and facilitators to advancement for women in academic surgery. JAMA Netw Open 2:e1910228

5. Wallis CJ, Ravi B, Coburn N, Nam RK, Detsky AS, Satkunasivam $R$ (2017) Comparison of postoperative outcomes among patients treated by male and female surgeons: a population based matched cohort study. BMJ 359:j4366

6. AAMC Data and Reports (2019). https://www.aamc.org/datareports. Accessed $6 \mathrm{Feb} 2020$

7. Jones CP (2000) Levels of racism: a theoretic framework and a gardener's tale. Am J Public Health 90:1212-1215

8. Pearson AR, Dovidio JF, Gaertner SL (2009) The nature of contemporary prejudice: insights from aversive racism. Soc Pers Psychol Compass 3:314-338

9. Wesson DE, King TE Jr, Todd RF et al (2006) Achieving diversity in academic internal medicine: recommendations for leaders. Am J Med 119:76-81

Publisher's Note Springer Nature remains neutral with regard to jurisdictional claims in published maps and institutional affiliations. 